Case Report

\title{
Cortical Blindness due to Bilateral Occipital Infarcts in a Renal Failure Patient with Prostate Cancer: A Rare Complication of Hemodialysis
}

\author{
O. G. Doluoglu, ${ }^{1}$ M. S. Saricaoglu, ${ }^{2}$ C. V. Oztekin, ${ }^{1}$ \\ A. Karakurt, ${ }^{2}$ A. O. Akdemir, ${ }^{1}$ and E. Koc $^{3}$ \\ ${ }^{1}$ Department of Urology II Clinic of Ankara Numune Education and Research Hospital, 06100 Ankara, Turkey \\ ${ }^{2}$ Department of Ophthalmology III Clinic of Ankara Numune Education and Research Hospital, 06100 Ankara, Turkey \\ ${ }^{3}$ Department of Nephrology Clinic of Ankara Numune Education and Research Hospital, 06100 Ankara, Turkey
}

Correspondence should be addressed to O. G. Doluoglu; drdoluoglu@yahoo.com.tr

Received 1 May 2012; Revised 8 November 2012; Accepted 20 December 2012

Academic Editor: Jonathan E. Sears

Copyright (c) 2013 O. G. Doluoglu et al. This is an open access article distributed under the Creative Commons Attribution License, which permits unrestricted use, distribution, and reproduction in any medium, provided the original work is properly cited.

Loss of vision is a rare complication seen in hemodialysis patients. It is thought to develop because of the hypotension that can be observed during dialysis. This paper involves a patient with acute loss of vision during hemodialysis due to bilateral occipital infarcts.

\section{Introduction}

Acute complications commonly occur during routine hemodialysis treatments. They include the following [1]: hypotension (25-55\%), cramps (5-20\%), nausea and vomiting (5$15 \%)$, headache $(5 \%)$, chest pain $(2-5 \%)$, back pain $(2-5 \%)$, itching $(5 \%)$, and fever and chills $(<1 \%)$.

Acute loss of vision can be observed in hemodialysis patients. It may be seen due to ischemic optic neuropathy (ION) or cerebral cortical infarcts caused by hypotension during the process. Cortical blindness is a very rare complication associated with hemodialysis, which to our best knowledge, is defined in only two reports in the literature $[2,3]$. This paper presents a patient with acute, bilateral, and complete loss of vision observed during hemodialysis, proved to be central and due to ischemic infarcts in the bilateral occipital lobes.

\section{Case Report}

A 65-year-old male patient presented to our institution with lower urinary tract symptoms and elevated serum urea and creatinine levels. He was under followup for type II diabetes mellitus for the last 6 years and prostate cancer for the last 3 years. Maximal androgen deprivation therapy ( $\mathrm{GnRh}$ analogue and nonsteroidal antiandrogen) was initiated 3 years ago, but the patient did not receive the treatment in a regular way. He had had 2 sessions of hemodialysis previously in emergency settings in another center. Blood tests at the time of first inspection were serum urea: $135 \mathrm{mg} / \mathrm{dL}$, creatinine: $6.2 \mathrm{mg} / \mathrm{dL}$, and prostate specific antigen: $>153 \mathrm{ng} / \mathrm{dL}$. Evaluation of the urinary tract revealed a maximal urinary flow rate of $6 \mathrm{~mL} / \mathrm{s}$, an international prostate symptom score (IPSS) of 29, and grade III dilation of bilateral kidneys and ureters on the sonogram. Invasion of the trigone and left ureteral orifice was observed, and right unit was double- $j$ stented during cystoscopy. Diversion of the left unit with a percutanous nephrostomy was recommended, but was refused by the patient. Transurethral resection of the prostate (TUR-P) was performed after serum creatinine was stabilized at a level of approximately $3 \mathrm{mg} / \mathrm{dL} 1$ month after stenting of the right collecting system. Patient was discharged with a serum creatinine level of $2.9 \mathrm{mg} / \mathrm{dL}$ and with conservative recommendations like increased oral hydration and lowprotein diet. The patient was meticulously followed after the TUR-P with monthly routine blood biochemistry and PSA 
measurements and urinary ultrasound. Four months later, the patient applied with a sudden onset tachycardia. Laboratory analysis revealed serum hemoglobin, total calcium, ionized calcium, urea, creatinine, potassium, and glucose levels of $8.7 \mathrm{~g} / \mathrm{dL}, 8.2 \mathrm{mg} / \mathrm{dL}, 4.7 \mathrm{mg} / \mathrm{dL}, 87 \mathrm{mg} / \mathrm{dL}, 3.61 \mathrm{mg} / \mathrm{dL}$, $7.2 \mathrm{mmol} / \mathrm{L}$, and $190 \mathrm{mg} / \mathrm{dL}$, respectively. Coagulation panel and urine analysis were normal. There were wide QT interval and upright $\mathrm{T}$ waves in the Electocardiogram (ECG). Nephrologist suggested infusion of $\mathrm{Ca}$ and initiation of hemodialysis by these findings. The baseline and predialysis blood pressures of the patients were $147 / 85 \mathrm{mmHg}$ and 150/90 $\mathrm{mmHg}$, respectively.

A dialysis duration of 2.5 hours was scheduled in order to achieve the target uro-reduction rate of $30 \% .1 .2 \mathrm{~m}^{2}$ synthetic biocompatible membrane (Fresenius F6 polisulfone low flux fully synthetic biocompatible membrane) was employed. Dialysate bicarbonate value was set to $30 \mathrm{mEq} / \mathrm{L}$. Heparin was not used as this was the first hemodialysis of the patient.

During dialysis, acute bilateral loss of vision developed in the patient and therefore the intervention was terminated. The highest and lowest systolic/diastolic blood pressures recorded during hemodialysis were $90 / 60$ and $80 / 50 \mathrm{mmHg}$, respectively.

Ophthalmological inspection revealed that patient's vision was at the hand-motion level, the light reflexes were normal, there was no afferent pupillar defect, bilateral posterior segments, and bilateral intraocular pressures were normal. Findings suggested a central pathology, and the CT scan performed afterwards revealed large hypodense infarct areas in the bilateral occipital and parasagittal posterior region of right parietal lobes (Figures 1 and 2). Bilateral carotid artery doppler and echocardiography for differential diagnosis of thromboembolism were normal.

Patient was conscious, cooperating, and well oriented, and all deep tendon reflexes were found to be hyperactive in the neurological evaluation. Also a few days later right hemiparesis appeared in the patient. Therefore patient was accepted in the intensive care unit of the neurology department and received two times of $0,6 \mathrm{cc}$ clexane and three times $10 \mathrm{mg}$ dexametasone a day. Thus our purpose was to protect the ischemic penumbra with anticoagulant and antiinflammatory effect.

Despite the recovery of hemiparesis, loss of vision continued during followup of the patient. Patient's loss of vision continued and did not recover throughout the followup.

\section{Discussion}

Renal Failure (RF) is loss of renal function best diagnosed and characterized with increased serum creatinine levels. It can be categorized into two major groups as acute and chronic RF. Acute RF can be further subgrouped into prerenal, intrinsic (renal), or postrenal RF's. Furthermore, acute RF can arise during the course of a chronic RF in $13 \%$ of the patients [4].

We hereby present a patient with RF due to severe bladder outlet obstruction together with the invasion of the trigone and obstruction of ureteral orifices, caused by prostate cancer. It was reported in a study that invasion of prostate cancer was

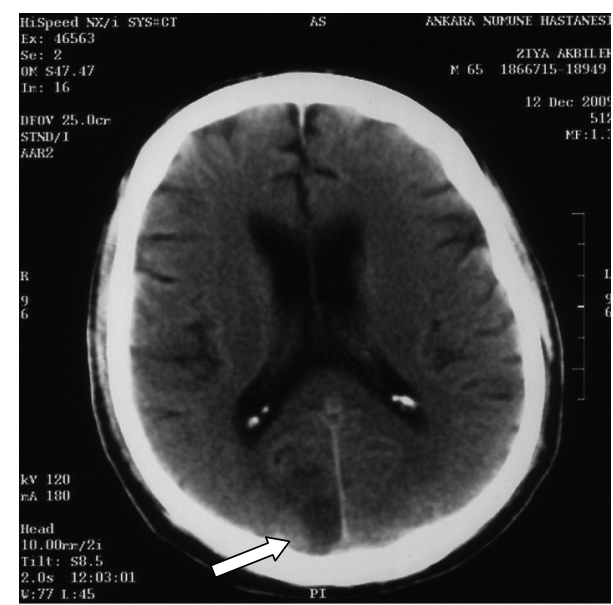

FIGURE 1: Right occipital infarct area in the CT scan (white arrow).

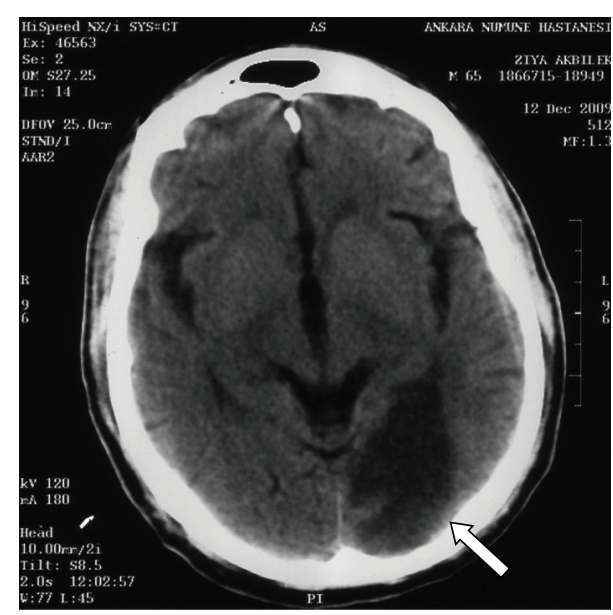

FIgURE 2: Left occipital infarct area in the CT scan (white arrow).

observed in lymph nodes, bones, lungs, and bladder, liver and adrenal in decreasing order in autopsy series [5].

Once RF develops, either acute or chronic, hemodialysis is always a treatment option on the table. Indications of hemodialysis include volume overload, severe hypercalcemia, severe metabolic acidosis, pericarditis, and other specific symptoms associated with azotemia $[6,7]$.

Loss of vision is a rare complication of hemodialysis which is thought to be associated with hypotension observed during the process [8]. On the other hand, hypotension is the most common complication of hemodialysis which is still observed in $20-30 \%$ of the patients despite the advances in medical technologies [9].

Loss of vision associated with hemodialysis may develop as a result of ION or cerebral cortical infarcts $[10,11]$. ION can either be anterior or posterior. Anterior ION may present with edema of the optic nerve and peripapillary hemorrhage. In the cases of posterior ION however, optic disc is always normal on examination $[8,12]$. Likewise in our case, pupillary reactions were positive and there was no afferent pupillary defect, and bilateral fundi were normal. 
These findings suggested a central pathology, most likely a cortical loss of vision due to occipital infarcts, which was later confirmed by the neuroradiological imaging.

The visual cortex is supplied by the posterior cerebral artery, and mainly its calcarinal branch. Also there is a rich anastomosis in the posterior pole, formed by the terminal branches of the posterior and middle cerebral arteries which is specifically important for the macular visual cortex. Vascular obliterations may cause loss of vision and impair visual field, generalized hypoperfusion of the cortex may result in severe loss of vision as is the case in our patient. Natural history of cortical blindness is unpredictable and yet to be fully understood as some may resolve spontaneously while others may not. The loss of vision in our patient was accepted to be irreversible as no improvements were observed in vision over time and his clinical status remained stable throughout the followup.

In order to prevent the visual complications during hemodialysis, changes in blood pressures of the patients during the process should be thoroughly monitored together with their general uremic and biochemical status; and any problem arising should be handled in a multidisciplinary approach including departments of urology, nephrology, and ophthalmology.

\section{References}

[1] H. Bregman, J. T. Daugirdas, and T. S. Ing, "Complications during hemodialysis," in Handbook of Dialysis, J. T. Daugirdas and T. S. Ing, Eds., p. 149, Little, Brown and Company, New York, NY, USA, 1994.

[2] Y. H. Al-Falki, "Bilateral occipital infarcts in a hemodialysis patient," American Journal of Nephrology, vol. 21, no. 5, pp. 413-414, 2001.

[3] D. I. Moel and Y. A. Kwun, "Cortical blindness as a complication of hemodialysis," Journal of Pediatrics, vol. 93, no. 5, pp. 890-891, 1978 .

[4] F. Liano and J. Pascual, "Epidemiology of acute renal failure: a prospective, multicenter, community-based study. Madrid acute renal failure study group," Kidney International, vol. 50, pp. 811-818, 1996.

[5] H. Saitoh, M. Hida, and T. Shimbo, "Metastatic patterns of prostatic cancer. Correlation between sites and number of organs involved," Cancer, vol. 54, no. 12, pp. 3078-3084, 1984.

[6] S. Pendse, A. Singh, and E. Zawada, "Initiation of dialysis," in Handbook of Dialysis, J. T. Daugirdas, P. G. Blake, and T. S. Ing, Eds., Lippincott Williams \& Wilkins, Philadelphia, Pa, USA, 4th edition, 2007.

[7] K/DOQI, “Clinical practice guidelines and clinical practice recommendations 2006 updates hemodialysis adequacy peritoneal dialysis adequacy vascular access," American Journal of Kidney Diseases, vol. 48, supplement 1, pp. S1-S105, 2006.

[8] K. S. Servilla and G. C. Groggel, "Anterior ischemic optic neuropathy as a complication of hemodialysis," American Journal of Kidney Diseases, vol. 8, no. 1, pp. 61-63, 1986.

[9] J. Feehally, J. Floege, and R. J. Johnson, "Comprehensive clinical nephrology," in Acute Complication of Hemodialysis, B. L. Jaber, A. Vpadhyay, and B. J. G. Pereira, Eds., chapter 84, pp. 967-969, 2007.
[10] S. Haider, N. J. Astbury, and D. V. Hamilton, "Optic neuropathy in uraemic patients on dialysis," Eye, vol. 7, no. 1, pp. 148-151, 1993.

[11] S. E. Connolly, K. B. Gordon, and J. C. Horton, "Salvage of vision after hypotension-induced ischemic optic neuropathy," American Journal of Ophthalmology, vol. 117, no. 2, pp. 235-242, 1994.

[12] L. M. Buono, R. Foroozan, P. J. Savino, H. V. Danesh-Meyer, and D. Stanescu, "Posterior ischemic optic neuropathy after hemodialysis," Ophthalmology, vol. 110, no. 6, pp. 1216-1218, 2003. 


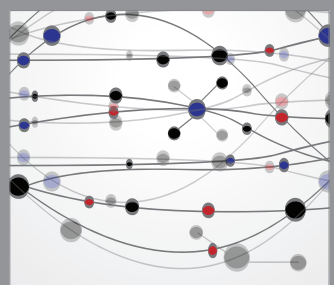

The Scientific World Journal
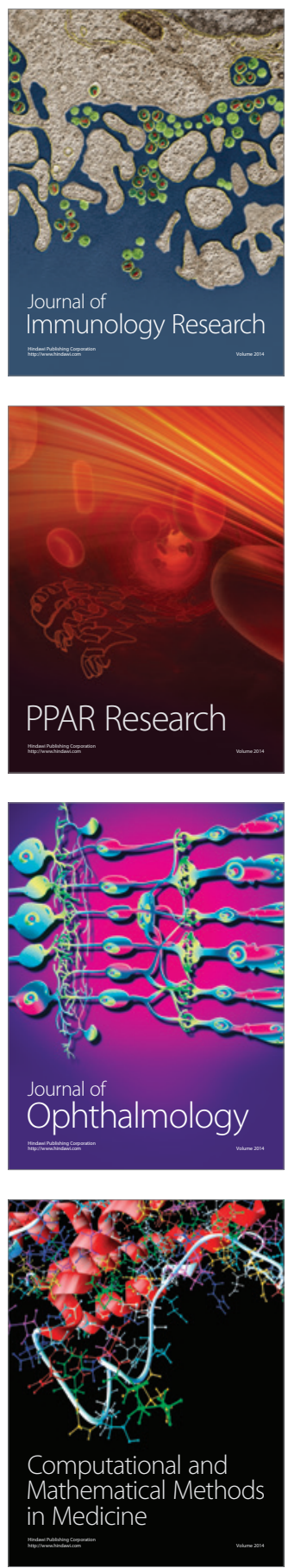

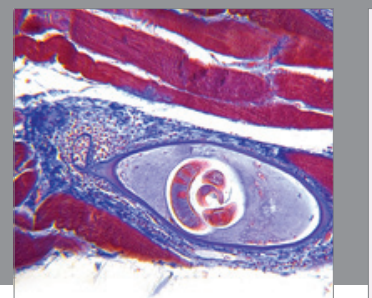

Gastroenterology

Research and Practice
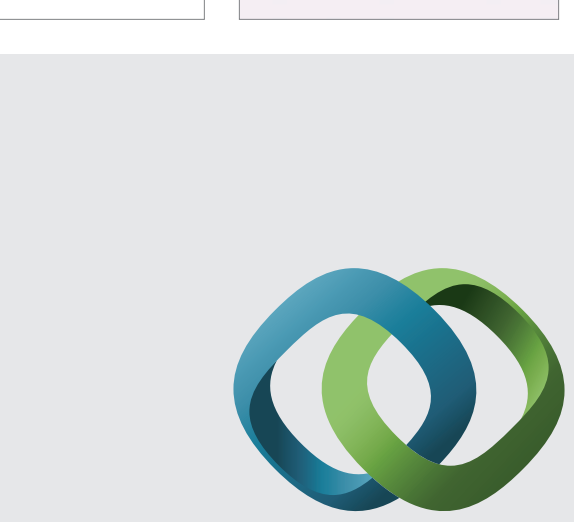

\section{Hindawi}

Submit your manuscripts at

http://www.hindawi.com
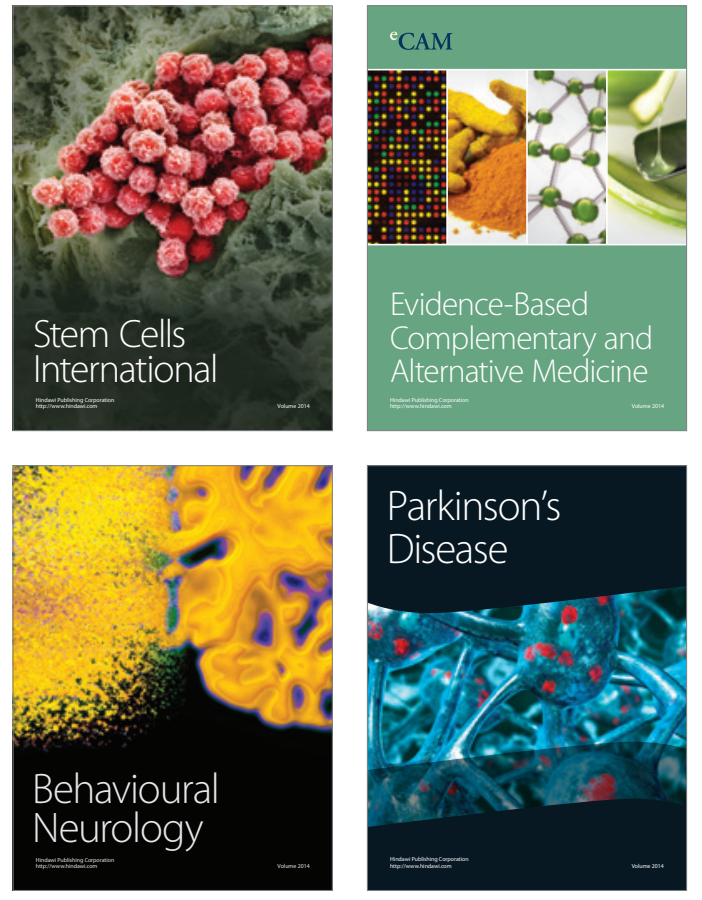
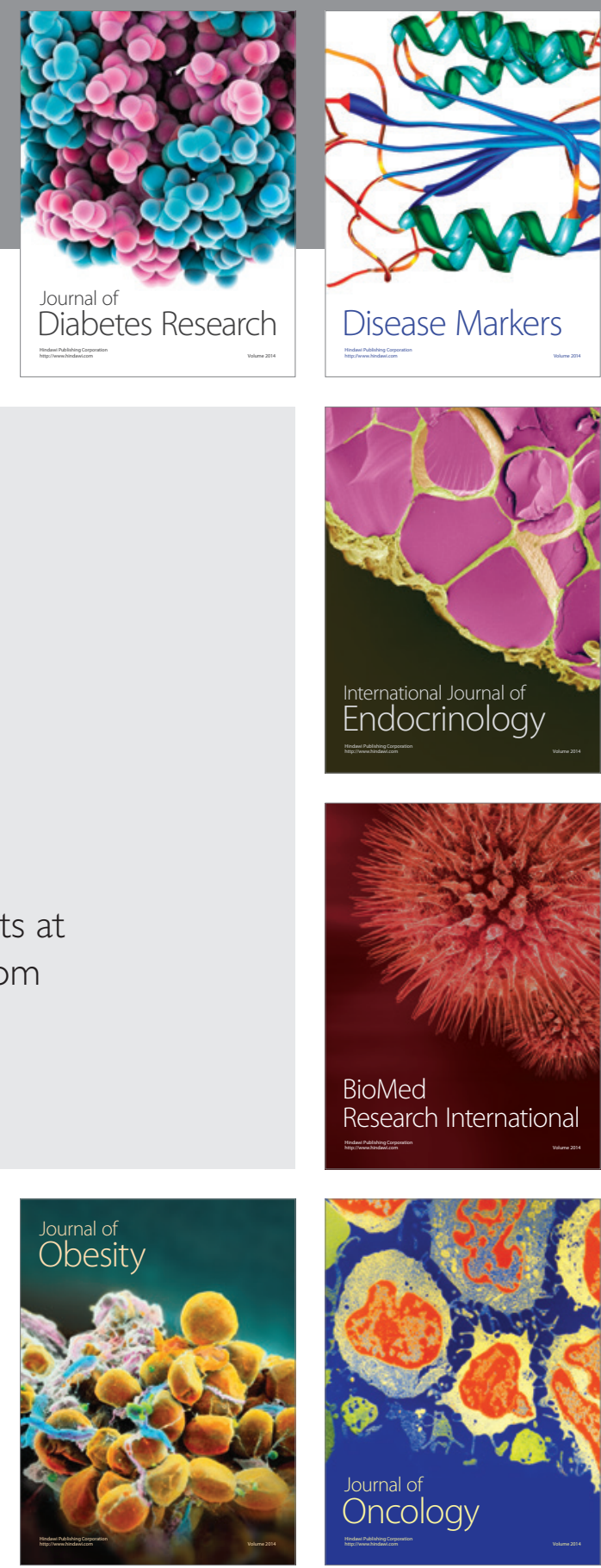

Disease Markers
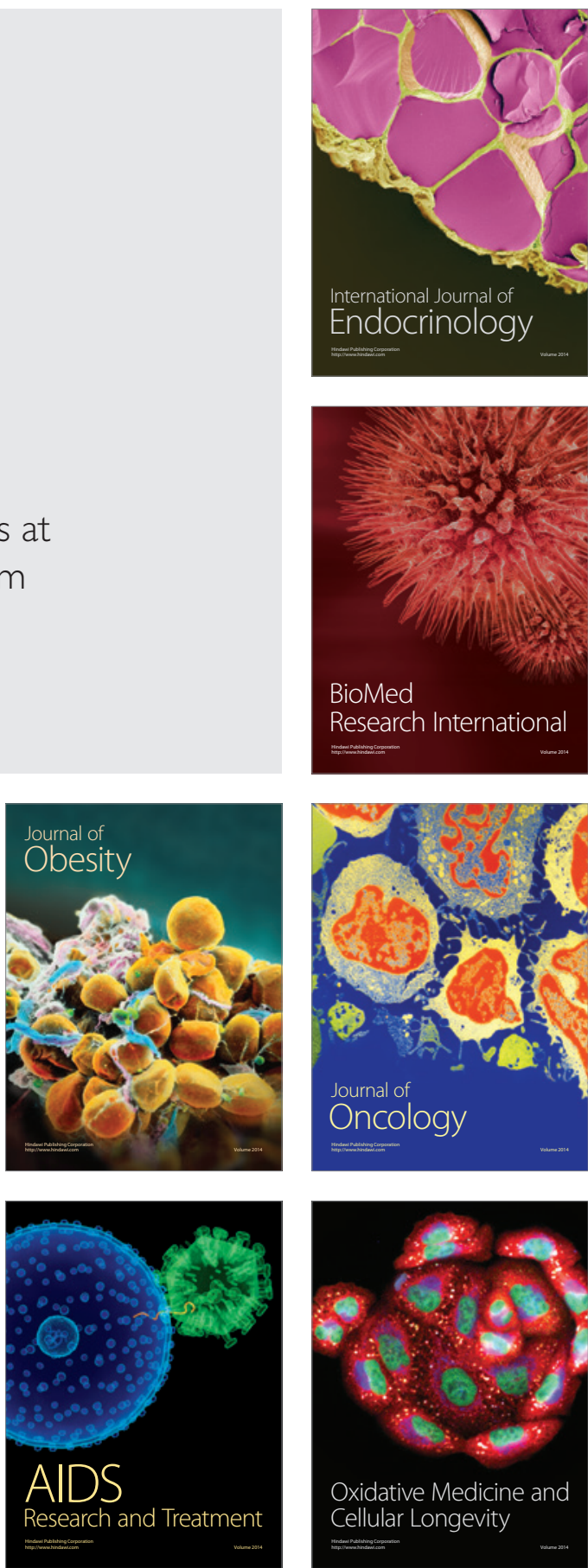